\title{
Freeze-thaw-induced changes of the zona pellucida explains decreased rates of fertilization in frozen-thawed mouse oocytes
}

\author{
J. Carroll, H. Depypere* and C. D. Matthews
}

Department of Obstetrics \& Gynaecology, University of Adelaide, The Queen Elizabeth Hospital, Woodville Road, Woodville, South Australia 5001, Australia; and ${ }^{*}$ Department of Obstetrics \& Gynaecology, University Hospital of Ghent, Ghent, Belgium

\begin{abstract}
Summary. Frozen-thawed oocytes have a reduced rate of fertilization $(48 \cdot 8 \%)$ when compared with unfrozen controls $(97 \%)$. In this study we have used zona-drilling to bypass the zona pellucida and investigate whether the decreased rate of fertilization is due to freezing-induced changes in the zona pellucida which prevent sperm penetration. After zona drilling the fertilization rate of frozen-thawed oocytes $(87 \cdot 8 \%)$ was the same as for zona-intact unfrozen controls ( $88 \%)$, indicating that freeze-thawinduced changes at the level of the zona pellucida were responsible for the decreased rate of fertilization.

To determine whether the changes were occurring during the manipulations before and after freezing or the complete freeze-thaw cycle, oocytes were exposed to the complete set of manipulations normally experienced during cryopreservation and appropriate control groups. A small but significant decrease in the rate of fertilization $(82.8 \%)$ was apparent in oocytes exposed to the manipulations before and after freezing compared with controls $(92 \cdot 2 \%)$. The freeze-thaw-induced changes in the zona pellucida therefore occur primarily during the complete freeze-thaw cycle itself and not the manipulations before and after freezing and are responsible for the decreased rate of fertilization observed in frozen-thawed oocytes.
\end{abstract}

Keywords: cryopreservation; oocyte; zona pellucida; in-vitro fertilization; mouse

\section{Introduction}

In-vitro fertilization and subsequent embryo transfer is an accepted treatment for human infertility. The advent of superovulation regimens and the high rate of fertilization obtained in vitro often result in the production of more embryos than can be safely returned to the donor immediately. Currently, surplus embryos are either frozen and transferred in a subsequent cycle or discarded. The low-temperature storage of human embryos has ethical and legal problems which are not associated with frozen gametes. The ability to store oocytes would also provide an opportunity of pregnancy to patients having undergone cancer treatment or who have had an oophorectomy at an early age. As a consequence there is currently considerable interest in a safe and efficient means of preserving the human oocyte. Except for the mouse (Whittingham, 1977) few successes in terms of live fetuses or birth have been reported after freezing of oocytes, although a few pregnancies have been reported for women (Chen, 1986; van Uem et al., 1987) and rabbits (Al-Hasani et al., 1989; Vincent et al., 1989).

The major problems associated with oocyte cryopreservation occur at the time of fertilization, namely an increased rate of polyploidy (Al-Hasani et al., 1987; Glenister et al., 1987; Carroll et al., 1989), shown in the mouse to be a result of digynic fertilization (Carroll et al., 1989), and a 
decreased rate of fertilization (Glenister et al., 1987; Carroll et al., 1989). Fertilization may be prevented at the zona pellucida, the vitelline membrane, or by failure of pronucleus formation and/ or syngamy. Our previous study (Carroll et al., 1989) showed that a decreased ability of spermatozoa to penetrate and form a pronucleus was largely responsible for the decrease in the fertilization rate of frozen-thawed oocytes. Two preliminary studies (Wood et al., 1988; Depypere et al., 1989) have implicated changes in the zona pellucida as a possible explanation for the decreased rate of fertilization but another study (Kola et al., 1988) has reported that although all frozen-thawed oocytes were fertilized the number that cleaved to the 2-cell stage was significantly reduced. In the present study, we have used zona drilling to breach the zona pellucida to investigate its role in the decreased rate of fertilization after freezing.

The cryopreservation procedure consists of 2 major components, the freeze-thaw cycle itself and the manipulations before and after freezing. These manipulations include cooling and the exposure of oocytes to cryoprotectants. Zona pellucida solubility, fertilization and spindle organization can be adversely affected by these processes (Johnson \& Pickering, 1987; Pickering \& Johnson, 1987; Johnson et al., 1988) but the degree of damage is clearly dependent upon the conditions in which oocytes are exposed (Johnson, 1989). We have exposed oocytes to the complete manipulations involved before and after freezing to determine their effect on oocyte fertilization and subsequent development.

The aim of this study, therefore, was to determine whether the zona pellucida is the site at which fertilization is inhibited and the component(s) of the freezing protocol responsible for the decreased rate of fertilization after freezing and thawing.

\section{Materials and Methods}

Source of oocytes. Mice, (C57 $\times$ CBA) F1, 3-6 weeks of age, were induced to superovulate by i.p. injections of 7.5 i.u. PMSG (Folligon: Intervet, Lyppard Chemicals, South Australia) and 7.5 i.u. hCG (Chorulon: Intervet) 48-52 $\mathrm{h}$ apart. The mice were killed 14-14.5 h after hCG and the oviducts were collected in Hepes-HTF (Quinn et al., 1985) supplemented with $5 \mathrm{mg}$ bovine serum albumin/ml (BSA: Fraction V: Sigma Chemical Co., St Louis, MO, USA). Cumulus masses were released into a solution of hyaluronidase $(300 \mathrm{IU} / \mathrm{ml}$ : bovine testis type IS H 3506 , Sigma) in the same medium and cumulus-free oocytes were washed in supplemented Hepes-HTF.

Freezing. The methods used for freezing and thawing were similar to those previously described by Whittingham et al. (1979). Cumulus-free oocytes were transferred to a dish containing 1.5 M-dimethylsulphoxide (DMSO: BDH Chemicals Ltd, Poole, Dorset, UK) in supplemented Hepes-HTF medium on ice. During this equilibration time $20-50$ oocytes were loaded into pre-cooled $\left(4^{\circ} \mathrm{C}\right) 0.25$ - $\mathrm{ml}$ plastic straws (IMV Victorian Artificial Breeders, Bacchus Marsh Victoria) containing the same medium. After equilibration for $10 \mathrm{~min}$ the loaded straws were placed in a Minicool Cell Freezer (Minicool AS25, France; supplier Liquid Air, Adelaide, South Australia) at $0^{\circ} \mathrm{C}$. The straws were cooled at $2^{\circ} \mathrm{C} / \mathrm{min}$ to $-7^{\circ} \mathrm{C}$. Ice formation was induced $1 \mathrm{~min}$ later by an automatic seeding device which was routinely monitored, and, when necessary, the straws were manually seeded using a pair of forceps prechilled in liquid nitrogen. At $5 \mathrm{~min}$ after seeding, straws were slowly cooled at $0.3^{\circ} \mathrm{C} / \mathrm{min}$ to $-40^{\circ} \mathrm{C}$, equilibrated for $15 \mathrm{~min}$ then rapidly chilled to $-110^{\circ} \mathrm{C}$. The straws were then transferred directly to liquid $\mathrm{N}_{2}$ and stored for periods between $12 \mathrm{~h}$ and 3 days.

Thawing and dilution of cryoprotectant. The oocytes were thawed rapidly $\left(2500^{\circ} \mathrm{C} / \mathrm{min}\right)$ by plunging the straws directly from liquid $\mathrm{N}_{2}$ into a water bath at $37^{\circ} \mathrm{C}$. As soon as thawing was complete the contents of the straws were released into $1.5 \mathrm{M}$-DMSO in Hepes-HTF supplemented with $5 \mathrm{mg} \mathrm{BSA} / \mathrm{ml}$ at room temperature and morphologically normal oocytes were collected. DMSO was diluted from these oocytes at room temperature in three 10-min equimolar steps ( $1.0 \mathrm{M}, 0.5 \mathrm{M}$ and Hepes-HTF). Oocytes which failed to rehydrate normally were discarded.

Zona drilling. The drilling procedure was carried out according to the methods of Gordon \& Talansky (1986). Briefly, oocytes were transferred to drops of supplemented Hepes-HTF overlaid with paraffin oil (liquid paraffin colourless, heavy: BDH). An oocyte was immobilized on the holding pipette and a drilling pipette ( $10 \mu \mathrm{m}$ in diameter) was used to apply a constant stream of acidified Tyrode's medium ( $\mathrm{pH} 2.5)$ to the zona pellucida until the zona was breached.

In-vitro fertilization and culture. Fertilization and culture was carried out in a bicarbonate-buffered HTF culture medium ( $\mathrm{HCO}_{3}-\mathrm{HTF}$; Quinn et al., 1985) containing $5 \mathrm{mg} \mathrm{BSA} / \mathrm{ml}$. Microdrops for culture and $0.5 \mathrm{ml}$ drops for fertilization were placed in plastic culture dishes (Falcon, Lincoln Park, NJ, USA), overlaid with oil and equilibrated 
overnight in an humidified atmosphere at $5 \% \mathrm{CO}_{2}, 5 \% \mathrm{O}_{2}$ and $90 \% \mathrm{~N}_{2}$ before use. All subsequent incubations and cultures were carried out in this atmosphere at $38-39^{\circ} \mathrm{C}$.

The detailed procedures for in-vitro fertilization of mouse ova have been described elsewhere (Quinn \& Whittingham, 1982). Briefly, each cauda epididymidis was removed from a mature $(\mathrm{C} 57 \times \mathrm{CBA}) \mathrm{F} 1$ male and placed in a fertilization dish (as above). Needles were used to release the spermatozoa into the medium which were allowed to disperse for $15 \mathrm{~min}$ in the incubator. The sperm concentration was then determined and a suitable volume added to the fertilization dishes, providing a final concentration of $2 \times 10^{6}$ spermatozoa $/ \mathrm{ml}$. The dishes were then incubated for $1-2 \mathrm{~h}$ to facilitate capacitation. Oocytes were washed in fresh culture medium and incubated with spermatozoa for $5 \mathrm{~h}$ before being removed, washed twice in fresh medium and transferred to microdrops for further culture. After $24 \mathrm{~h}$ the number of 2-cell embryos was determined and the number of expanded blastocysts was assessed after $90 \mathrm{~h}$ of culture.

Experiment 1. To determine the effect of the zona pellucida on the fertilization rate of frozen-thawed oocytes zona-drilling was carried out on a group of fresh and frozen-thawed oocytes. Two zona-intact control groups were used, one as a routine control for in-vitro fertilization in which the oocytes were fertilized immediately after collection and another which served to control for the time taken to carry out the zona-drilling procedure ( $40-50 \mathrm{~min})$. Oocytes of this group were placed in drops of supplemented Hepes-HTF under oil and kept at $37^{\circ} \mathrm{C}$ and fertilized concomitantly with the zona-drilled group. To test whether zona-drilling caused parthenogenetic activation of frozen-thawed oocytes groups of zona-drilled fresh and frozen-thawed oocytes were cultured in microdrops as described above and examined after $8 \mathrm{~h}$ for the presence of pronuclei and after $24 \mathrm{~h}$ for cleavage to the 2 -cell stage.

Experiment 2. Oocytes were collected and cumulus cells removed. After washing, the oocytes were randomly divided into 5 groups designed to assess the effects of ageing, cooling and cryoprotectant experienced during a freezing programme on the fertilization rate and development of oocytes. All incubations were carried out in supplemented Hepes-HTF. The groups were: (1) immediately fertilized after collection; (2) exposed to complete manipulations before and after freezing as previously described (i.e. $10 \mathrm{~min}$ in $1.5 \mathrm{M}$-DMSO at $0^{\circ} \mathrm{C}$ then warmed to room temperature for $5 \mathrm{~min}$ before dilution of the DMSO in 3 equimolar steps at room temperature); (3) $10 \mathrm{~min}$ at $0^{\circ} \mathrm{C}$; (4) $10 \mathrm{~min}$ at $0^{\circ} \mathrm{C}$ followed by $40 \mathrm{~min}$ at room temperature; and (5) room temperature for $50 \mathrm{~min}$.

\section{Results}

\section{Morphological survival after freezing and thawing}

The majority of oocytes were recovered from the freezing straws $(98 \%)$. Before removal of the cryoprotectant $78 \%$ were considered to be morphologically normal. After removal of the cryoprotectant, $65 \%$ of the oocytes remained intact.

\section{Effect of cryopreservation on fertilization and development}

In both zona-intact control groups the fertilization rate of frozen-thawed oocytes was approximately half that of the unfrozen controls (Fig. 1; $P<0.0005$ ). The delay in insemination times in the two control groups produced a small but non-significant reduction in fertilization rate. Fewer 2-cell embryos derived from frozen-thawed oocytes developed to the blastocyst stage in each of the 3 treatments (Fig. 2; $P<0.05$ ).

\section{Effect of zona drilling on fertilization and development}

Zona-drilled fresh $(n=40)$ and frozen-thawed $(n=40)$ oocytes incubated in the absence of spermatozoa showed no evidence of pronuclei formation or cleavage to the 2-cell stage. After insemination significantly more zona-drilled frozen-thawed oocytes cleaved to the 2-cell stage than did frozen-thawed zona-intact controls (Fig. 1; $P<0.0005$ ). After zona drilling the fertilization rate of frozen-thawed oocytes was similar to that of zona-intact unfrozen controls (Fig. 1; $P>0.05)$. A small (11\%) but significant difference remained between zona-drilled fresh and frozen oocytes (Fig. $1 ; P<0 \cdot 01$ ).

The ability of embryos to form blastocysts was not affected by zona drilling before or after freezing (Fig. 2; $P>0.05$ ). 


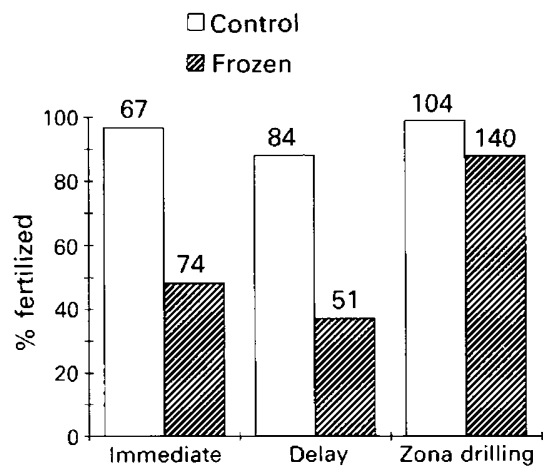

Fig. 1. The effect of cryopreservation on oocytes fertilized immediately after recovery, after a delay, and after zona drilling. Results are from 2 experiments. The number at the top of each column represents the total number of oocytes inseminated.

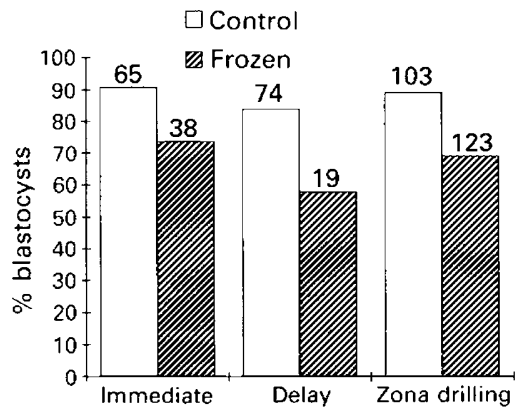

Fig. 2. The in-vitro development of 2-cell embryos derived from fresh and frozen-thawed oocytes. Results are from 2 experiments. The number at the top of each column represents the total number of 2-cell embryos cultured.

\section{Effect of manipulations before and after freezing on fertilization and development}

The fertilization rate of oocytes in Group 2 was approximately $4-12 \%$ lower than that in the other treatment and control groups (Table 1). This difference was significant when compared to oocytes from Groups 1, 3 and 5 but not from Group 4. The fertilization rate of oocytes from Group 4 was similar to all other groups.

In-vitro development to blastocysts was significantly better in Group $1(P<0.05)$ and Group 3 $(P<0.005)$ oocytes when compared with those in Group 2 (Table 1). All other treatments were not significantly different.

\section{Discussion}

Freezing and thawing reliably reduced the fertilization rate of mouse oocytes (Glenister et al., 1987; Carroll et al., 1989; present study). Zona drilling is a procedure which uses acidified Tyrode's medium to breach the zona pellucida, and has been shown to overcome conditions in which the fertilization rate is compromised, including reduced numbers of spermatozoa (Gordon \& Talansky, 1986; Depypere et al., 1988) and oocytes matured in vitro (Vanderhyden et al., 1989). In this study we have used zona drilling to investigate whether freeze-thaw-induced changes in the zona pellucida are responsible for the decreased rate of fertilization after freezing. Although no increase 
Table 1. The effect of manipulations before and after freezing on fertilization and development of mouse oocytes

\begin{tabular}{lccc}
\hline Treatment & $\begin{array}{c}\text { No. of } \\
\text { oocytes }\end{array}$ & $\begin{array}{c}\text { No. of 2-cell } \\
\text { embryos }(\%)\end{array}$ & $\begin{array}{c}\text { No. of blastocysts* } \\
(\%)\end{array}$ \\
\hline (1) Immediately fertilized & 153 & $141^{\mathrm{b}}(92 \cdot 2)$ & $120^{\mathrm{b}}(85 \cdot 1)$ \\
(2) $\begin{array}{l}\text { Manipulations before } \\
\text { and after freezing }\end{array}$ & 116 & $96^{\mathrm{a}}(82 \cdot 8)$ & $60^{\mathrm{a}}(62 \cdot 5)$ \\
(3) $10 \mathrm{~min}$ at $0^{\circ} \mathrm{C}$ & 90 & $87^{\mathrm{b}}(96 \cdot 7)$ & $74^{\mathrm{b}}(85 \cdot 1)$ \\
(4) $\quad 10 \mathrm{~min}$ at $0^{\circ} \mathrm{C}+40 \mathrm{~min}$ & 91 & $83^{\mathrm{ab}}(91 \cdot 2)$ & $61^{\mathrm{ab}}(73 \cdot 5)$ \\
$\quad$ at room temperature & 126 & $119^{\mathrm{b}}(94 \cdot 4)$ & $87^{\mathrm{ab}}(73 \cdot 1)$ \\
\hline
\end{tabular}

The results from 6 individual experiments were combined and analysed by $\chi^{2}$ tests.

Treatments in a column with different superscripts are significantly different, $P<0.05$.

*Expressed as \% of 2-cell embryos.

in the rate of parthenogenetic activation has previously been reported it is apparent that care must be taken when exposing oocytes to acidified Tyrode's medium (Johnson et al., 1990). Our results showed no evidence of parthenogenetic activation after the zona drilling of fresh or frozen-thawed oocytes, indicating that increased rates of cleavage are due to the events of fertilization. Zonadrilling increased the fertilization rate of frozen-thawed oocytes to the same level as for zona-intact unfrozen controls, demonstrating that the reduced rate of fertilization after freezing and thawing was due to freezing-induced changes at the level of the zona pellucida. We have previously shown that failure of spermatozoa to penetrate the oocyte and/or form a pronucleus is a major contributory factor to the decreased rate of fertilization after cryopreservation (Carroll et al., 1989). This was supported in another study using a different freezing procedure which showed high rates of sperm penetration in frozen-thawed zona-free oocytes compared with zona-intact oocytes (Wood et al., 1988). The present results are similar, suggesting that the block to fertilization is at the zona pellucida rather than the oocyte membrane or cytoplasm. However, Kola et al. (1988) obtained a high fertilization rate of frozen-thawed oocytes but only about half of these cleaved to the 2-cell stage. The explanation for the difference in these results is not clear but it may be that the method for assessing fertilization was different in the latter study or was detrimental to subsequent cleavage.

Cryopreservation can be divided into two components, the freeze-thaw cycle itself and the manipulations before and after freezing. In this study we have assessed the extent to which the exact manipulations used before and after freezing in an established freezing protocol affect the rate of fertilization and development of oocytes (Table 1). The results show a small but significant decline in the fertilization rate when oocytes are exposed to the complete set of manipulations when compared with appropriate control groups, except when oocytes were cooled to $0^{\circ} \mathrm{C}$ for $10 \mathrm{~min}$ and kept at room temperature for $40 \mathrm{~min}$. Cooling and exposure of mouse oocytes to DMSO have been shown to increase zona pellucida resistance to chymotrypsin, but this is clearly dependent upon the conditions of exposure (Johnson et al., 1988). Addition of DMSO at $4^{\circ} \mathrm{C}$ proved to be the protocol least deleterious (Johnson, 1989) and is similar to the protocol used in previous successful cryopreservation programmes (Whittingham, 1977; Chen, 1986; Carroll et al., 1989). In the present study the reduced rate of fertilization after exposure to the manipulations before and after freezing $(\sim 10 \%)$ was clearly not as marked as that induced by the complete freezing procedure $(\sim 50 \%)$. This demonstrates that during cryopreservation under optimal conditions the major changes in the zona pellucida which limit fertilization occur during the complete freeze-thaw cycle and are not the result of the manipulations before and after freezing.

The developmental capacity of oocytes exposed to the complete set of manipulations before and after freezing was not different from that of frozen-thawed oocytes but was less than for non- 
manipulated controls. This indicates that the reduced developmental capacity of frozen-thawed oocytes in the present study may be a result of the manipulations before and after freezing. Further, since none of the control groups (3-5; Table 1) showed a significant decline in developmental capacity, it appears that exposure to DMSO and/or its interaction with one or more of the other variables (time, temperature) may explain the reduced developmental capacity after exposure to the manipulations before and after freezing.

The nature of the freeze-thaw-induced changes at the zona pellucida is not clear. After fertilization or activation the zona pellucida becomes impenetrable to spermatozoa and resistant to digestion by chymotrypsin (Inoue \& Wolf, 1974, 1975). This is believed to be a result of the release of cortical granules from the oocyte (Gulyas, 1980, for review) with resultant modification and/or cross linking of the zona pellucida glycoproteins (Wassarman, 1990, for review). However, a number of events not associated with fertilization or activation can also result in increased chymotrypsin resistance and a corresponding decrease in fertilization, e.g. ageing and in-vitro maturation of germinal vesicle stage oocytes (Wolf \& Hamada, 1976; DeFelici \& Siracusa, 1982; Gianfortoni \& Gulyas, 1985). Cortical granule-associated fucosylated glycoconjugates have been shown to be absent from the surface of frozen-thawed oocytes (Wood et al., 1988), indicating that the extensive cortical granule exocytosis which occurs at fertilization (Lee et al., 1988) was not apparent after freezing. This does not preclude the possibility of a slow release of cortical granules or release of a population of cortical granules not stained by the lectins used. The presence of serum is known to prevent zona hardening during the in-vitro maturation of oocytes (Downs et al.,1986; Choi et al., 1987) and we have observed a similar effect on frozen-thawed oocytes (Wood et al., 1989). The presence of serum (Ducibella et al., 1990) has also been shown to prevent the conversion of the zona pellucida protein responsible for interacting with acrosome-reacted spermatozoa, ZP2, to its inactive form $\mathrm{ZP}_{\mathrm{f}}$ (Bleil et al., 1988), thus providing a possible mechanism for the zona hardening response in the absence of fertilization. Morphological observations on frozen-thawed oocytes which have failed to fertilize illustrate large numbers of spermatozoa firmly bound to the zona pellucida, indicating that the zona-pellucida sperm receptor is apparently not affected by cryopreservation.

It must also be considered that structural changes in the zona pellucida not previously associated with the zona reaction or zona hardening may occur during freezing. The zona pellucida is a highly porous acellular coat which consists of an organized network of glycoproteins (Greve \& Wassarman, 1985). A structure of this nature is likely to be directly affected by freezing-associated effects such as dehydration and ice crystal growth which may, therefore, offer a simple explanation for the freezing-induced changes of the zona pellucida. Clearly, further work is required to determine whether the freezing-induced modifications at the zona pellucida are elicited via oocytemediated events such as cortical granule release or a direct effect of freezing and thawing on the zona pellucida itself.

We thank Dr S. Flaherty for helpful comments on the manuscript; Mr J. McLaughlin for micromanipulation pipettes; and Mrs Helen Holmes for typing the manuscript.

\section{References}

Al Hasani, S., Diedrich, K., van der Ven, H., Reiniekce, A., Hartje, M. \& Krebs, D. (1987) Cryopreservation of human oocytes. Human Reprod. 2, 696-700.

Al-Hasani, A., Kirsch, J., Diedrich, K., Blanke, S., van der Ven, H. \& Krebs, D. (1989) Successful embryo transfer of cryopreserved and in vitro fertilized rabbit oocytes. Human Reprod. 4, 77-79.

Bleil, J.D., Greve, J.M. \& Wassarman, P.M. (1988) Identification of a secondary sperm receptor in the mouse egg zona-pellucida: role in maintenance of binding of acrosome reacted sperm to eggs. Devl Biol. 128, 376-385.

Carroll, J., Warnes, G.M. \& Matthews, C.D. (1989) Increase in digyny explains polyploidy after in-vitro fertilization of frozen-thawed mouse oocytes. $J$. Reprod. Fert. 85, 489-494.

Chen, C. (1986) Pregnancy after human oocyte cryopreservation. Lancet 1, 884-886.

Choi, T.S., Mori, M., Kohmoto, K. \& Shoda, Y. (1987) Beneficial effect of serum on the fertilizability of mouse 
oocytes matured in vitro. J. Reprod. Fert. 79, 505-508.

DeFelici, M. \& Siracusa, G. (1982) "Spontaneous" hardening of the zona pellucida of mouse oocytes during in vitro culture. Gamete Res. 6, 107-113.

Depypere, H.T., McLaughlin, K.J., Seamark, R.F., Warnes, G.M. \& Matthews, C.D. (1988) Comparison of zona-cutting and zona-drilling as techniques for assisted fertilization in the mouse. J. Reprod. Fert. 84, 205-211.

Depypere, H.T., Carroll, J.C., Matthews, C.D. \& Vanderkerckhove, D. (1989) Zona drilling improves the in vitro fertilization rate of cooled and frozenthawed oocytes. Proc. Europ. Soc. Hum. Reprod. Embryol. 103, abstr.

Downs, S.M., Schroeder, A.C. \& Eppig, J.J. (1986) Serum maintains the fertilizability of mouse oocytes matured in vitro by preventing hardening of the zona pellucida. Gamete Res. 15, 115-122.

Ducibella, T., Kurasawa, S., Rangarajan, S., Kopf, G.S. \& Schultz, R.M. (1990) Precocious loss of cortical granules during mouse oocyte meiotic maturation and correlation with an egg-induced modification of the zona-pellucida. Devl Biol. 137, 46-55.

Gianfortoni, J.G. \& Gulyas, B.J. (1985) The effects of short term incubation (aging) of mouse oocytes on in vitro fertilization, zona solubility and embryonic development. Gamete Res. 11, 59-216.

Glenister, P.H., Wood, M.J., Kirby, C. \& Whittingham, D.G. (1987) Incidence of chromosome anomalies in first-cleavage mouse embryos obtained from frozenthawed oocytes fertilized in vitro. Gamete Res. 16, 205-216.

Gordon, J.W. \& Talansky, B.E. (1986) Assisted fertilization by zona-drilling: a mouse model for the correction of oligospermia. J. exp. Zool. 239, 347-354.

Greve, J.M. \& Wassarman, P.M. (1985) Mouse egg extracellular coat is a matrix of interconnected filaments possessing a structural repeat. J. med. Biol. 181, 253-264.

Gulyas, B.J. (1980) Cortical granules of mammalian eggs. Int. Rev. Cytol. 63, 357-392.

Inoue, M. \& Wolf, D.P. (1974) Comparative solubility properties of the zona pellucida of unfertilized and fertilized mouse ova. Biol. Reprod. 11, 558-565.

Inoue, M. \& Wolf, D.P. (1975) Fertilization-associated changes in the murine zona pellucida: a time sequence study. Biol. Reprod. 13, 546-551.

Johnson, M.H. (1989) The effect on fertilization of exposure of mouse oocytes to dimethyl sulphoxide: An optimal protocol. J. In Vitro Fert. \& Embryo Transf. 6, 168-175.

Johnson, M.H. \& Pickering, S.J. (1987) The effect of dimethylsulphoxide on the microtubule system of the mouse oocyte. Development 100, 313-324.

Johnson, M.H., Pickering, S.J. \& George, M.A. (1988) The influence of cooling on the properties of the zona pellucida of the mouse oocyte. Hum. Reprod. 3, $383-387$.
Johnson, M.H., Pickering, S.J., Braude, P.R., Vincent, C., Cant, A. \& Currie, J. (1990) Acid Tyrode's solution can stimulate parthenogenetic activation of human and mouse oocytes. Fert. Steril. 53, 266-270.

Kola, I., Kirby, C., Shaw, J., Davey, A. \& Trounson, A. (1988) Vitrification of mouse oocytes results in aneuploid zygotes and malformed fetuses. Teratology 38, 467-474.

Lee, S.H., Ahuja, K.K., Gilburt, D.J. \& Whittingham, D.G. (1988) The appearance of glycoconjugates associated with cortical granule release during mouse fertilization. Development 102, 595-604.

Pickering, S.J. \& Johnson, M.H. (1987) The influence of cooling on the organisation of the meiotic spindle of the mouse oocyte. Hum. Reprod. 2, 207-216.

Quinn, P. \& Whittingham, D.G. (1982) Effect of fatty acids on fertilization and development of mouse embryos in vitro. J. Androl. 3, 440-444.

Quinn, P., Kerin, J.F. \& Warnes, G.M. (1985) Improved pregnancy rate in human in vitro fertilization with the use of a medium based on the composition of human tubal fluid. Fert. Steril. 44, 493-498.

Vanderhyden, B.C., McLaughlin, K.J., Rutledge, J.M. \& Armstrong, D.T. (1989) Zona drilling increases the penetrability of rat oocytes matured in vitro. Biol. Reprod. 40, 953-960.

van Uem, J.F.H.M., Siebzehnrubl, E.R., Schuh, B., Koch, R., Trotnow, S. \& Lang, N. (1987) Birth after cryopreservation of unfertilized oocytes. Lancet ii, $752-753$.

Vincent, C., Garnier, J., Heyman, Y. \& Renard, J.P. (1989) Solvent effects on cytoskeletal organization and in-vivo survival after freezing of rabbit oocytes. J. Reprod. Fert. 87, 809-820.

Wassarman, P.M. (1990) Profile of a mammalian sperm receptor. Development $108,1-17$.

Whittingham, D.G. (1977) Fertilization in vitro and development to term of unfertilized mouse oocytes previously stored at $-196^{\circ} \mathrm{C}$. J. Reprod. Fert. 49, 8994.

Whittingham, D.G., Wood, M., Farrant, J., Lee, H. \& Halsey, J.A. (1979) Survival of frozen mouse embryos after rapid thawing from $-196^{\circ} \mathrm{C} . J$. Reprod. Fert. 56, 11-21.

Wolf, D.P. \& Hamada, M. (1976) Age dependent losses in the penetrability of mouse eggs. J. Reprod. Fert. 48 , 213-214.

Wood, M.J., Lee, S-H. \& Whittingham, D.G. (1988) Oocyte freezing inhibits subsequent sperm penetration of the zona pellucida. J. Reprod. Fert., Abstr. Ser. 1, Abstr. 13.

Wood, M.J., Carroll, J. \& Whittingham, D.G. (1989) The addition of serum to the medium limits zona hardening in frozen oocytes. J. Reprod. Fert. Abstr. Ser. 3, Abstr. 56.

Received 13 March 1990 